\title{
Sistemas estaduais de avaliação: interfaces com qualidade e gestão da educação
}

\author{
State assessment systems: interface with quality and education management \\ Sistemas estatales de evaluación: interfaz con la calidad y gestión de la \\ educación
}

\section{CRISTIANE MACHADO \\ OCIMAR MUNHOZ ALAVARSE \\ PAULO HENRIQUE ARCAS}

Resumo: Explorar possíveis interfaces entre a configuração dos Sistemas Estaduais de Avaliação com qualidade e gestão da educação é o objetivo deste artigo. Dentre os 27 entes federados, 19 possuem sistemas próprios de avaliação (LOPES, 2007; SOUSA; OLIVEIRA, 2010; GAME, 2011). Verificou-se que, de modo geral, esses sistemas possuem as mesmas características e o mesmo desenho do Sistema de Avaliação da Educação Básica (Saeb). Constataram-se as tendências de uso dos dados obtidos para subsidiar a elaboração de indicadores estaduais de qualidade da educação e dos resultados das aferições dos estudantes para avaliação de desempenho docente, com a atribuição de gratificações e bônus, indicando a adoção da lógica de que a produção da qualidade e a gestão da educação podem ser forjadas com base em mecanismos de quase mercado.

Palavras-chave: Qualidade; gestão da educação; sistemas de avaliação.

\begin{abstract}
To explore possible interfaces between the configuration of the State Assessment Systems quality and management of education is the goal of this article. Among the 27 federal agencies, 19 have their own evaluation systems (LOPES, 2007; SOUSA and OLIVEIRA, 2010; GAME, 2011). It was found that, in general, these systems have the same features and design of the Basic Education Evaluation System (SAEB). It found the data usage trends obtained to support the development of state indicators of quality of education and the results of measurements of students to evaluate teaching performance, with the attribution of gratifications and bonuses, indicating the adoption of the logic that quality production and management education could be forged based on mechanisms of nearly market.
\end{abstract}

Keywords: Quality; Management of education; Assessment systems.

Resumen: Explorar posibles interfaces entre la configuración de los Sistemas Estatales de Evaluación con la calidad y gestión de la educación es el objetivo de este artículo. Entre las 27 agencias federales, 19 tienen sus propios sistemas de evaluación (LOPES, 2007; SOUSA y OLIVEIRA, 2010; GAME, 2011). Se verificó que, en general, esos sistemas poseen las mismas características y el diseño del Sistema de Evaluación de la Educación Básica (SAEB). Se constató las tendencias de uso de datos obtenidos para apoyar el desarrollo de indicadores estatales de calidad de la educación y de los resultados de las mediciones de 
los estudiantes para evaluar el desempeño docente, con la atribución de gratificaciones y bonos, indicando la adopción de una lógica de que la producción de la calidad y la gestión de la educación pueden ser forjadas sobre la base de mecanismos de casi mercado.

Palabras clave: Calidad, Gestión de la educación, Sistemas de evaluación.

\section{INTRODUÇÃO}

A Conferência de Jomtien, em 1990, foi um marco não só no debate sobre educação no mundo como também na mudança da organização e proposição de iniciativas e ações educativas. Após esse evento, é possível observar, com certa regularidade, duas características que se destacam nas políticas educacionais da maioria dos países. Por um lado, há um visível consenso de que a educação escolar é condição indispensável para o desenvolvimento econômico das sociedades, principalmente nos países considerados emergentes e, por outro, é notória a crescente hegemonia de implantação de sistemas de avaliação pautados na aferição do desempenho dos estudantes em provas padronizadas como mecanismo de gestão e melhoria da qualidade da educação (ADAMS, ACEDO: POPA,2012).

Nesse contexto, o governo federal criou, também em 1990, o Sistema de Avaliação da Educação Básica (Saeb), desencadeando um movimento de ênfase na implantação de iniciativas de avaliação de sistema como instrumento de gestão das políticas educacionais e melhoria da educação. Essa medida impulsionou a proposição de políticas semelhantes pelos entes federados, principalmente os estados. Assim, em 2014, é possível identificar a existência de Sistemas Estaduais de Avaliação em 19 estados brasileiros dentre os 27 entes federados, sendo 26 estados e o Distrito Federal, conforme pesquisas desenvolvidas e publicadas (LOPES, 2007; SOUSA; OLIVEIRA, 2010; GAME, 2011) e dados recentes coletados por meio de consultas às páginas das Secretarias de Estado da Educação, do Centro de Políticas Públicas e Avaliação da Educação (CAEd), da Universidade Federal de Juiz de Fora, e de notícias veiculadas em jornais na internet.

Com base nessa constatação, o objetivo deste trabalho é evidenciar possíveis aproximações com qualidade e gestão da educação a partir da configuração que os Sistemas Estaduais de Avaliação vêm adotando. O texto retoma, inicialmente, alguns estudos sobre os contornos das iniciativas de avaliação de sistema dos estados brasileiros para, nas considerações finais, analisar possíveis interfaces entre as características das avaliações dos estados com qualidade e gestão educacional. 


\section{CONTORNOS DOS SISTEMAS ESTADUAIS DE AVALIAÇÃO}

Os estados brasileiros foram implantando seus próprios sistemas de avaliação gradativamente e, em geral, seguiram o desenho original do Saeb como inspiração para sua formatação. Investigando o tema, Bonamino, Bessa e Franco (2004) afirmam que se percebe que

A interação com o INEP/MEC especialmente o SAEB, tem papel fundamental no movimento dirigido para a consolidação de sistemas estaduais de avaliação, além de contribuir diretamente para a implementação da metodologia e da tecnologia para a construção e análise dos programas e medidas educacionais (p. 77).

Ainda nos anos 1990, iniciativas de sistemática de avaliação própria começaram a ser esboçadas em alguns estados, como Ceará e Minas Gerais, em 1992, Paraná, em 1995 e São Paulo, em1996, embora, especialmente nos casos de Minas Gerais e Paraná, a constituição dos sistemas de avaliação com as características atuais se deu somente nos anos 2000, período no qual se verifica ampla profusão dessas iniciativas pelos estados brasileiros (ALAVARSE; BRAVO; MACHADO, 2012).

Pesquisa realizada pelo Grupo de Avaliação e Medidas Educacionais da Faculdade de Educação da Universidade Federal de Minas Gerais (Game/ FAE/UFMG, 2011) registra as seguintes justificativas dos gestores para a criação de sistemas de avaliação: demora na divulgação dos resultados do Saeb; caráter amostral do Saeb, que limitava o desenvolvimento de políticas que pudessem atender às especificidades de redes e escolas, e a necessidade de promover avaliações anuais, já que o Saeb era aplicado a cada dois anos. Para os autores:

\footnotetext{
A expansão das avaliações subnacionais parece refletir justamente a necessidade de controlar mais detidamente as etapas que envolvem o processo de avaliação e, assim o fazendo, traçar um diagnóstico mais contextual da aprendizagem dos alunos para, com base nisso, elaborar instrumentos e estratégias talvez mais eficazes (GAME/FAE/UFMG, 2011, p. 08).
}

O desdobramento do Saeb, em 2005, em duas avaliações, sendo uma delas censitária, conhecida como Prova Brasil, não arrefeceu o ímpeto dos estados na implantação de seus próprios sistemas de avaliação, embora alguns questionamentos tenham sido evidenciados, como apontam Sousa e Oliveira (2010): 
Quando o governo federal, unilateralmente, toma a iniciativa de realizar uma testagem censitária, a Prova Brasil, apresentam-se dois tipos de problemas. De um lado, os estados passam a questionar-se acerca da validade da manutenção de sistemas próprios, com seus altos custos e complexidade logística, para realizar a mesma função que o governo federal já realiza. De outro, se estabelece uma frequência muito alta de aplicação de provas, o que inviabiliza, até mesmo, o tempo necessário para que seus resultados sejam absorvidos, analisados e transformados em ações por parte das redes de ensino (p. 812).

Apesar disso, o que se nota é o fortalecimento dos sistemas já existentes e o surgimento de sistemas em estados que até então não haviam tido experiências de avaliação do seu sistema de ensino ou em que estas eram muito incipientes.

Em 2007, outra mudança empreendida pelo governo federal, a criação do Índice de Desenvolvimento da Educação Básica (Ideb), poderia representar a possibilidade de alinhamento dos sistemas estaduais com as avaliações e o índice federais, diminuindo a importância das avaliações estaduais, ou mesmo estimulando a extinção de alguns dos sistemas de avaliação estaduais. Porém, o movimento que se observou foi não só de continuidade dos sistemas estaduais como, também, a criação de indicadores próprios em alguns estados.

O reconhecimento da ampliação de iniciativas de sistemas estaduais de avaliação impeliu os pesquisadores a estudá-los. Algumas pesquisas procuraram mapear e caracterizar os sistemas de avaliação estaduais e aprofundar aspectos relacionados aos usos dos resultados obtidos por esses sistemas de avaliação no planejamento e formulação de políticas educacionais.

Dentre essas pesquisas, destacamos estudo realizado por Sousa e Oliveira (2010) sobre os sistemas de avaliação de cinco estados brasileiros; o doutoramento de Lopes (2007), que buscou mapear e analisar os sistemas de avaliação existentes em todos os estados brasileiros, e a investigação conduzida por pesquisadores do Grupo de Avaliação e Medidas Educacionais (GAME), da Faculdade de Educação da Universidade Federal de Minas Gerais (UFMG). Além dessas, é importante mencionar o mapeamento das iniciativas de implantação de sistemas de avaliação estaduais elaborado por Oliveira (2012), do Centro de Políticas Públicas e Avaliação da Educação (CAEd) da Universidade Federal de Juiz de Fora (UFJF).

A pesquisa realizada por Sousa e Oliveira (2010) teve o "propósito de caracterizar e cotejar sistemas de avaliação por cinco unidades federadas (Bahia, Ceará, Minas Gerais, Paraná e São Paulo), buscando compreender seus pressupostos e contornos bem como analisar se e como os resultados produzidos têm informado a formulação e implementação de suas políticas educacionais" (p. 795). Os sistemas foram analisados considerando suas características durante os anos de 2005 a 2007. 
O estudo de Lopes (2007) identificou a existência de 16 estados que haviam criado, até o momento de sua pesquisa, sistemas próprios de avaliação. Quando concluída a pesquisa, 14 dos 16 sistemas identificados ainda estavam em vigência. A investigação feita pelo GAME (2011) revelou a existência de sistemas de avaliação em 18 estados. Já o mapeamento feito por Oliveira (2012) encontrou 19 sistemas estaduais de avaliação da educação básica.

Em estudo recente, realizado em fevereiro de 2015, foi feita uma atualização do mapeamento da situação de existência dos sistemas estaduais de avaliação. Esse levantamento indicou que, entre os 26 estados e o Distrito Federal, somente sete unidades da federação não possuem sistemas de avaliação: Amapá, Distrito Federal, Maranhão, Mato Grosso, Rio Grande do Norte, Santa Catarina e Sergipe. Cabe ressaltar que, em alguns estados, as avaliações de sistemas foram paralisadas, até mesmo extintas em alguns casos, porém retomadas posteriormente, como é o caso do estado do Paraná. Além disso, é importante destacar que alguns estados que atualmente não possuem sistemas de avaliação estão anunciando sua criação, como, por exemplo, vem ocorrendo no estado do Maranhão. No Quadro 1, são apresentados os estados que, com base nesse levantamento, possuem sistema de avaliação, adicionalmente com informações sobre ano de criação e última aplicação de seus instrumentos.

Quadro 1 - Sistemas Estaduais de Avaliação. 1992-2014

\begin{tabular}{|c|c|c|c|c|c|}
\hline & Estado & Nome & Sigla & $\begin{array}{l}\text { Ano de } \\
\text { criação }\end{array}$ & $\begin{array}{c}\text { Última } \\
\text { Aplicação }\end{array}$ \\
\hline 01 & Acre & $\begin{array}{c}\text { Sistema Estadual de Avaliação da } \\
\text { Aprendizagem Escolar }\end{array}$ & SEAPE & 2009 & 2012 \\
\hline 02 & Alagoas & $\begin{array}{l}\text { Sistema de Avaliação Educacional de } \\
\text { Alagoas/ Avaliação de Aprendizagem da } \\
\text { Rede Estadual de Educação de Alagoas }\end{array}$ & SAVEAL/ AREAL & 2001 & 2012 \\
\hline 03 & Amazonas & $\begin{array}{l}\text { Sistema de Avaliação do Desempenho } \\
\text { Educacional do Amazonas }\end{array}$ & SADEAM & 2008 & 2013 \\
\hline 04 & Bahia & Sistema de Avaliação Baiano de Educação & SABE & 2010 & 2013 \\
\hline 05 & Ceará & $\begin{array}{l}\text { Sistema Permanente de Avaliação da } \\
\text { Educação Básica do Ceará }\end{array}$ & SPAECE & 1992 & 2014 \\
\hline 06 & Espírito Santo & $\begin{array}{c}\text { Programa de Avaliação da Educação Básica } \\
\text { do Espírito Santo }\end{array}$ & PAEBES & 2000 & 2014 \\
\hline 07 & Goiás & $\begin{array}{c}\text { Sistema de Avaliação Educacional do } \\
\text { Estado de Goiás }\end{array}$ & SAEGO & 2011 & 2013 \\
\hline 08 & $\begin{array}{l}\text { Mato Grosso } \\
\text { do Sul }\end{array}$ & $\begin{array}{c}\text { Sistema de Avaliação da Educação da Rede } \\
\text { Pública de Mato Grosso do Sul }\end{array}$ & SAEMS & 2003 & 2014 \\
\hline 09 & Minas Gerais & $\begin{array}{l}\text { Sistema Mineiro de Avaliação da Educação } \\
\text { Pública }\end{array}$ & SIMAVE & 2000 & 2014 \\
\hline 10 & Pará & Sistema Paraense de Avaliação Educacional & SisPAE & 2013 & 2014 \\
\hline 11 & Paraíba & Sistema de Avaliação da Educação da Paraíba & Não possui & 2011 & 2014 \\
\hline
\end{tabular}


Quadro 1 - Sistemas Estaduais de Avaliação. 1992-2014

\begin{tabular}{|c|c|c|c|c|c|}
\hline & Estado & Nome & Sigla & $\begin{array}{c}\text { Ano de } \\
\text { criação }\end{array}$ & $\begin{array}{c}\text { Última } \\
\text { Aplicação }\end{array}$ \\
\hline 12 & Paraná & $\begin{array}{c}\text { Sistema de Avaliação da Educação } \\
\text { Básica do Paraná }\end{array}$ & SAEP & 2012 & 2013 \\
\hline 13 & Pernambuco & $\begin{array}{c}\text { Sistema de Avaliação da Educação } \\
\text { Básica de Pernambuco }\end{array}$ & SAEPE & 2000 & 2014 \\
\hline 14 & Piauí & $\begin{array}{c}\text { Sistema de Avaliação Educacional do } \\
\text { Piauí }\end{array}$ & SAEPI & 2011 & 2014 \\
\hline 15 & Rio de Janeiro & $\begin{array}{c}\text { Sistema de Avaliação da Educação do } \\
\text { Estado do Rio de Janeiro }\end{array}$ & SAERJ & 2008 & 2014 \\
\hline 16 & Rio Grande do Sul & $\begin{array}{c}\text { Sistema Estadual de Avaliação } \\
\text { Participativa / } \\
\text { Sistema de Avaliação do Rendimento } \\
\text { Escolar do Rio Grande do Sul }\end{array}$ & SAERS & $\begin{array}{c}2012 \\
(\text { SEAP) }\end{array}$ & 2013 \\
\hline 17 & Rondônia & $\begin{array}{c}\text { Sistema de Avaliação Educacional de } \\
\text { Rondônia }\end{array}$ & SAERO & 2012 & 2013 \\
\hline 18 & São Paulo & $\begin{array}{c}\text { Sistema de Avaliação do Rendimento } \\
\text { Escolar do Estado de São Paulo }\end{array}$ & SARESP & 1996 & 2014 \\
\hline 19 & Tocantins & $\begin{array}{c}\text { Sistema de Avaliação da Educação do } \\
\text { Estado do Tocantins }\end{array}$ & Salto & 2011 & 2014 \\
\hline
\end{tabular}

As informações colhidas mostram que os sistemas estaduais de avaliação da educação basicamente se caracterizam pela aplicação de testes padronizados aos estudantes da educação básica (ensino fundamental e ensino médio) e questionários contextuais, respondidos por gestores, professores, pais e alunos. Em geral, os testes padronizados envolvem as áreas de língua portuguesa e matemática; porém alguns sistemas têm ampliado as áreas avaliadas para ciências da natureza e ciências humanas, assim como criado avaliações específicas para alfabetização, caso dos estados de Bahia, Ceará, Goiás, Espírito Santo, Minas Gerais, Rondônia e São Paulo.

Outro aspecto a se considerar é que, a exemplo do governo federal, os governos estaduais, utilizando os resultados dos seus sistemas de avaliação, vêm criando também índices de qualidade da educação; casos dos estados do Amazonas, com o Índice do Desenvolvimento da Educação no Amazonas (IDEAM), Espírito Santo com o Indicador de Desenvolvimento das Escolas do Espírito Santo (IDE), Goiás, com o Índice de Desenvolvimento da Educação Básica do Estado de Goiás (IDEGO), Paraíba com o Índice de Desempenho da Educação da Paraíba (IDEPB), Pernambuco com o Índice de Desenvolvimento da Educação Básica de Pernambuco (IDEPE) e São Paulo com o Índice de Desenvolvimento da Educação do Estado de São Paulo (IDESP). A criação de indicadores de qualidade pelos estados, seguindo a política adotada pelo governo federal, indica ser esta forte tendência nas políticas educacionais em curso no país. 
O que se vislumbra, com base nesses estudos, é que o Saeb vem consolidando-se como referência de política de avaliação da Educação Básica, uma vez que esse sistema é visto como modelo indutor de iniciativas semelhantes pelos estados. Assim, conforme ponderam Alavarse, Bravo e Machado (2012), "essas iniciativas foram efetuadas na esteira do fortalecimento da avaliação como diretriz da política educacional no governo federal tanto no que se refere ao tempo histórico, como também à forma constituinte dos sistemas” (p. 6-7).

Analisando eventuais usos dos resultados das avaliações externas com esta lógica, Machado e Alavarse (2014) são contundentes ao afirmarem que

\begin{abstract}
Não estão afastados os efeitos deletérios da adoção de políticas de avaliação, notadamente quando ou transferem responsabilidades de gestores para professores ou se assentam em condições precárias de realização que, por sua vez, comprometem a própria qualidade de seus resultados (431-432).
\end{abstract}

Os estudos consultados e o levantamento recentemente feito corroboram a importância e o crescente uso da avaliação externa como mecanismo de aferição do desempenho dos estudantes em provas padronizadas e a tradução desses resultados em qualidade da educação. Alguns estudiosos, porém, indicam limitações na concepção de qualidade da educação baseada preponderantemente na proficiência dos alunos. Mesmo reconhecendo, assim como Casassus (2007), que "a qualidade da educação, como objetivo, tornou-se um conceito estratégico nas formulações de política educacional na grande maioria dos países" (p. 41), é preocupante a tendência de compreensão de qualidade na/da educação apenas pelos dados obtidos com as avaliações externas. Assim como assevera Dourado (2007), qualidade é "um fenômeno complexo, abrangente e que envolve múltiplas dimensões” (p. 9), não podendo ser associada a uma ou outra dimensão somente da educação e, muito menos, do trabalho desenvolvido nas escolas.

Mesmo se considerarmos o Índice de Desenvolvimento da Educação Básica (Ideb), criado em 2007 para ser um indicador da qualidade da educação nacional, que agrega dados de aprovação com os resultados dos alunos na Prova Brasil, ainda assim, não dá conta da complexidade do fenômeno educativo. Vianna (1990) já alertava para a necessidade de se considerarem outras dimensões da educação para se avaliar sua qualidade, reiterando que

A avaliação da qualidade da educação não se limita apenas à verificação do rendimento escolar, que é um momento na caracterização dessa qualidade. O desempenho dos estudantes em pesquisas da qualidade da educação é melhor compreendido e interpretado quando se levantam informações sobre o tipo de ensino que recebem, os procedimentos que vivenciam em sala de aula e no colégio, ainda sobre as características ambientais da família que determinam os seu comportamento (p. 99).

RBPAE - v. 31, n. 3, p. 667 - 680 set./dez. 2015 • 673 
Parte das informações que Vianna (1990) indica podem ser levantadas nos questionários contextuais aplicados juntamente com as provas. Ao que parece, esses dados são pouco divulgados e não se explicita como têm servido para subsidiar uma melhor leitura do desempenho dos alunos e tampouco se e como são utilizados no planejamento e formulação de políticas educacionais.

Neste sentido, Minhoto (2013) ressalta que

É preciso criar a cultura de avaliar a qualidade dos sistemas de ensino tendo em vista um número maior de variáveis que não apenas aquela gerada pela boa aferição do que os alunos sabem e são capazes de fazer e com isso evitar a incoerência de transferir as contradições sociais do plano objetivo para o plano subjetivo (p. 141).

A centralidade que a avaliação veio assumindo na gestão dos sistemas de educação e também na gestão das escolas tem gerado críticas principalmente por serem resultados dos sistemas de avaliação utilizados como indicadores da qualidade, como aponta Freitas (2013):

No que tange à qualidade, parece-nos que a avaliação tem sido utilizada como a redentora de todos os males da educação, transformando-se em um fim em si mesma. Há uma ilusão social de que avaliar os sistemas garante qualidade. Entende-se que aumentar a proficiência dos estudantes nos exames é o mesmo que elevar a qualidade, sendo esta medida somente por meio de indicadores e dados. Conceito polissêmico tanto do ponto de vista pedagógico, quanto social e político, a qualidade da educação não pode ser compreendida de forma descolada da historicidade do termo, favorecendo uma maneira superficial de entendimento e uso do mesmo (p. 167).

No entanto, vale sublinhar que não é possível conceber qualidade da educação sem considerar que desempenho dos alunos seja elemento relevante. Assim, com base em Machado e Alavarse (2014), advoga-se que

Se a qualidade na educação é um fenômeno complexo que possui determinações intraescolares, tais como currículo, formação docente, gestão escolar, avaliação da aprendizagem, condições de trabalho, infraestrutura das escolas etc., e extraescolares, tais como condições de vida da população, capital econômico, cultural e social das famílias dos alunos, entorno social da escola, distribuição de renda, violência, entre outros, o aumento do desempenho dos alunos nos exames é parte importante desse fenômeno, ainda que este não se esgote naquele, pois a medição da aprendizagem permite o aprofundamento do diagnóstico da situação da educação brasileira e o delineamento de iniciativas de políticas educacionais nesse terreno (p. 429-430). 
A Lei Federal no 13.005 de 25 de junho de 2014, que aprova o Plano Nacional de Educação (2014-2024), ressalta a relevância da obtenção de informações sobre a qualidade da educação para informar as políticas públicas e orienta para a constituição de um Sistema Nacional de Avaliação da Educação Básica baseado em indicadores de rendimento escolar e em:

II - indicadores de avaliação institucional, relativos a características como o perfil do alunado e do corpo dos (as) profissionais da educação, as relações entre dimensão do corpo docente, do corpo técnico e do corpo discente, a infraestrutura das escolas, os recursos pedagógicos disponíveis e os processos da gestão, entre outras relevantes (artigo 11 , parágrafo $1^{\circ}$ ).

Lopes (2007) buscou, em sua pesquisa de doutorado, pesquisar também iniciativas de avaliação do desempenho docente e de avaliação institucional implementadas pelas secretarias estaduais de educação. $O$ estudo indicou serem, naquele momento, muito incipientes essas iniciativas, encontrando a existência de quatro sistemas ou programas de avaliação do desempenho docente nos estados de Goiás, Sergipe, Rio de Janeiro e Tocantins, e apenas três estados que realizavam avaliação institucional: Acre, Ceará e Paraná.

Sousa e Oliveira (2010) destacam iniciativas estaduais, como é o caso do Ceará, que vislumbravam a necessidade de se ampliar a concepção de sistema de avaliação, nela incluindo e integrando outras dimensões a serem avaliadas:

\footnotetext{
A partir de 2000, o Spaece contemplou tanto a avaliação em larga escala quanto a avaliação institucional que, até então, vinha sendo realizada como atividade paralela ao Spaece. Considerando as características dos delineamentos adotados nos estados em análise, a configuração que assume a sistemática de avaliação no Ceará parece a mais inovadora no sentido de articular diversas vertentes, dimensões e fluxos ao processo avaliativo (p. 808).
}

A pesquisa de Sousa e Oliveira (2010, p. 816) evidenciou, também, que vêm sendo ampliados os usos que os estados têm feito dos resultados das avaliações, para além da referência de qualidade da educação. Os autores detectaram nas políticas de avaliação dos estados analisados que "uma das iniciativas que já se esboça é a articulação da avaliação de desempenho dos alunos a carreira, remuneração (incluindo gratificações e bônus) e mesmo à certificação docente" (p. 816).

Essa constatação encontra eco em análise de Bonamino e Sousa (2012), que, examinando a trajetória das políticas avaliativas na educação básica no país, descrevem três gerações de avaliações em larga escala, as de segunda e terceira geração aquelas que associam "políticas de responsabilização baseadas em 
consequências simbólicas e materiais têm o propósito de criar incentivos para que o professor se esforce no aprendizado dos alunos" (p. 383).

Esta tendência também foi observada por Santos, Nogueira, Jesus e Cruz (2012) em estudo que recolheu informações através da consulta aos sites das Secretarias de Estado da Educação para mapear a existência de políticas de avaliação do desempenho docente nas redes estaduais. O levantamento identificou que, dos 27 estados da federação, 16 possuíam, no momento em que se realizou o estudo, processos de avaliação do desempenho docente. No Quadro 2, é apresentada uma síntese dos achados das pesquisadoras.

\section{Quadro 2-Estados e Política de Avaliação do Desempenho Docente}

\begin{tabular}{|c|c|c|c|}
\hline \multicolumn{2}{|r|}{ Estado } & Política de avaliação do desempenho docente & Ano de criação \\
\hline 01 & Acre & Prêmio Anual de Valorização e Desenvolvimento Profissional & 2010 \\
\hline 02 & Amazonas & Premiação por Mérito do Desempenho Educacional & 2008 \\
\hline 03 & Bahia & $\begin{array}{l}\text { Avaliação do desempenho docente por meio de provas, sem } \\
\text { nome específico, relacionada à progressão funcional }\end{array}$ & 2009 \\
\hline 04 & Ceará & Prêmio Aprender para Valer & 2009 \\
\hline 05 & Espírito Santo & Bônus Desempenho & 2011 \\
\hline 06 & Goiás & $\begin{array}{c}\text { Bonificação docente: Programa Reconhecer - Estímulo à } \\
\text { Regência }\end{array}$ & 2011 \\
\hline 07 & Minas Gerais & $\begin{array}{l}\text { Avaliação de Desempenho Individual (ADI), instituída pela Lei } \\
\text { Complementar } n^{0} 71 / 2003\end{array}$ & 2003 \\
\hline 08 & Paraíba & Prêmio Educação Exemplar & 2011 \\
\hline 09 & Paraná & $\begin{array}{l}\text { Programa de Desenvolvimento Educacional (PDE) - programa de } \\
\text { formação continuada inserido no Plano de Carreira do Magistério }\end{array}$ & 2007 \\
\hline 10 & Pernambuco & $\begin{array}{l}\text { Bonificação docente estruturada a partir da Lei de } \\
\text { Responsabilidade Educacional e dos resultados do SAEPE e } \\
\text { IDEPE }\end{array}$ & 2008 \\
\hline 11 & Rio de Janeiro & $\begin{array}{l}\text { Programa para o aprimoramento e valorização dos servidores } \\
\text { públicos da Secretaria de Educação - instituído pelo Decreto nº } \\
42.793 / 2011\end{array}$ & 2011 \\
\hline 12 & $\begin{array}{l}\text { Rio Grande do } \\
\text { Norte }\end{array}$ & $\begin{array}{l}\text { Lei Complementar } n^{0} 322 / 06 \text { regulamenta a progressão funcional, } \\
\text { mas não estruturada na prática }\end{array}$ & 2006 \\
\hline 13 & Roraima & Prêmio Professor Excelência & 2011 \\
\hline 14 & São Paulo & $\begin{array}{c}\text { Sistema de Promoção para os Integrantes do Quadro do } \\
\text { Magistério - Lei Complementar 1.097/2009 }\end{array}$ & 2009 \\
\hline 15 & Sergipe & $\begin{array}{c}2004 \text { - Sistema de Avaliação Periódica de Desempenho } \\
\text { (Saped) do profissional do magistério. Gratificação Variável de } \\
\text { Desempenho (Gravad) } \\
2011 \text { - "Índice Guia" - proposta segundo a qual as escolas } \\
\text { precisam atingir as metas estabelecidas pela Secretaria de } \\
\text { Educação a partir das avaliações externas (Provinha Brasil, } \\
\text { Prova Brasil e Enem) }\end{array}$ & 2011 \\
\hline 16 & Tocantins & Prêmio de Valorização da Educação Pública do Tocantins & 2011 \\
\hline
\end{tabular}

Fonte: Santos, Nogueira, Jesus, Cruz (2012) 
Investigando a centralidade que a avaliação tem assumido na formulação das políticas educacionais em todos os níveis, Machado (2012) sopesa que "no caso da educação básica, a avaliação externa vem, também, paulatinamente ultrapassando as cercanias das escolas, estreitando a distância entre o avaliador (governo) e o avaliado (escola) e produzindo referenciais nacionais de qualidade de ensino" (p. 72).

No entanto, Machado (2013) chama a atenção para o fato de que

\begin{abstract}
As avaliações empreendidas pelos governos estaduais estão obtendo um lugar privilegiado na relação com as escolas e com a gestão das mesmas por se localizarem geográfica e colaborativamente mais próximas que o governo federal e suas ações. Porém, cabe ressaltar que essa proximidade, justamente por ela, pode, também, provocar efeitos colaterais deletérios como iniciativas de burla dos resultados, insegurança no fornecimento das informações e dados confidenciais e reações de animosidade da comunidade pelos resultados alcançados pela escola de seus filhos (p. 49).
\end{abstract}

Portanto, mesmo se tratando de um movimento com diferenciações, nos alcances e ritmos, o que se demarca nacionalmente é como a ampla difusão de iniciativas estaduais de sistemas de avaliação vem se configurando como um dos principais elementos das políticas educacionais na perspectiva de mecanismo de gestão e melhoria da qualidade na educação.

\title{
CONSIDERAÇÕES FINAIS
}

O movimento de incremento da avaliação externa como mecanismo de gestão da educação e instrumento de melhoria da qualidade do ensino vem, cada vez mais, expandindo-se e se fortalecendo, apontando para um crescente uso dessas iniciativas por governos estaduais, tanto como referência de qualidade como instrumento de gestão da educação.

A concepção de qualidade que se destaca é pautada pelo resultado das proficiências dos estudantes em provas padronizadas, como se somente essa dimensão resumisse todo o trabalho escolar. Como já acentuado, nos primórdios das proposições avaliativas em educação básica se asseverava que o desempenho dos alunos, para ser compreendido, deveria ser contextualizado com outras informações, tais como aquelas referentes às condições da escola, ao processo pedagógico, ao clima escolar, ao nível socioeconômico, entre outras.

O direcionamento do uso dos resultados da avaliação externa pelos governos como mecanismo de gestão se consolida paulatinamente. O desdobramento do Saeb, em 2005, na Prova Brasil é um dos fatores que nos permite tal dedução. No caso das avaliações estaduais, a natureza das relações políticas 
estabelecidas entre o órgão central gestor e as escolas pode favorecer o uso da avaliação como instrumento de gestão dos sistemas e das unidades escolares.

Observa-se, também, forte tendência, a partir dos anos 2000, de uso dos resultados das avaliações dos alunos como mecanismo de avaliação de desempenho dos docentes e, em alguns casos, como referencial para atribuição de gratificações e bônus. Constatação que aponta para a aceitação de uma concepção de ação estatal fundamentada numa lógica de gestão da educação baseada na classificação e premiação de escolas e alunos mediante a comparação das proficiências destes últimos, configurando um tipo de gestão forjada com base em mecanismos de quase mercado.

Ao finalizar, cabe reafirmar a centralidade que a avaliação, principalmente as conduzidas pelos estados brasileiros, tem tido na referência de qualidade e de gestão da educação. No entanto, salienta-se ser imprescindível o cuidado de que as avaliações externas sejam colocadas a serviço de uma educação pública de qualidade para todos os estudantes, sem quaisquer distinções, base de uma sociedade verdadeiramente democrática.

\section{REFERÊNCIAS}

ADAMS, Don; ACEDO, Clementina; POPA, Simona. In search of quality education. In: ADAMS, Don; ACEDO, Clementina; POPA, Simona. (Ed.). Quality and qualities: tensions in education reforms. Rotterdam: Sense; Paris: Unesco: International Bureau of Education, 2012. (Comparative and International Education: a Diversity of Voices, v. 16). p. 1-22.

ALAVARSE, Ocimar M.; BRAVO, Maria Helena; MACHADO, Cristiane. Avaliação como diretriz das Políticas Educacionais dos Governos Federal, Estaduais e Municipais: o caso brasileiro. Trabalho apresentado no III Congresso Ibero Americano de Política e Administração da Educação. Zaragoza, Espanha. 2012.

BONAMINO, Alicia; BESSA, Nícia; FRANCO, Creso. O "estado da avaliação" nos Estados. In: Bonamino, A.C., Bessa, N., Franco, C. (Org.) A avaliação da educação básica: pesquisa e gestão. Rio de Janeiro: Editora PUC-Rio; São Paulo: Loyola, 2004, p. 65-78,

BONAMINO, Alicia; SOUSA, Sandra Maria Zákia Lian. Três gerações de avaliação da educação básica no Brasil: interfaces com o currículo da/na escola. Educação e Pesquisa, São Paulo, v. 38, n. 2, p. 373-388, abr./jun. 2012. 
BRASIL. Presidência da República. Plano Nacional de Educação-PNE. Lei Federal 13.005 de 25 de junho de 2014. Disponível em: <http://www.planalto. gov.br/ccivil_03/_Ato2011-2014/2014/Lei/L13005.htm>. Acesso em: 25 mar. 2015.

CASASSUS, Juan. A escola e a desigualdade. Brasília:Líber Livro, 2007.

DOURADO, Luiz Fernando (Org.). A Qualidade da Educação: conceitos e definições. Brasília: MEC/Inep, 2007.

FREITAS, Luiz Carlos. Caminhos da avaliação de Sistemas Educacionais no Brasil: o embate entre a cultura da auditoria e a cultura da avaliação. In: BAUER, Adriana; GATTI, Bernadete A.; TAVARES, Marialva R. (Org.). Vinte e cinco anos de avaliação de sistemas educacionais no Brasil: implicações nas redes de ensino, no currículo e na formação de professores. Florianópolis: Insular, 2013. p. 147-176.

GAME (Grupo de avaliação e medidas educacionais). A avaliação externa como instrumento da gestão educacional nos estados: relatório final. Belo Horizonte: Game/ Faculdade de Educação / Universidade Federal de Minas Gerais, ago. 2011.

LOPES, Valéria. V. Cartografia da avaliação educacional no Brasil. Tese (Doutorado) - Faculdade de Educação, Universidade de São Paulo, São Paulo, 2007.

MACHADO, Cristiane; ALAVARSE, Ocimar Munhoz. Qualidade das escolas: tensões e potencialidades das avaliações externas. Educação \& Realidade, Porto Alegre, v. 39, n. 2, p. 413-436, abr./jun. 2014.

MACHADO, Cristiane. Impactos da avaliação externa nas políticas de gestão educativa. Revista Iberoamericana sobre Calidad, Eficacia y Cambio en Educación, v. 11, n. 1. p. 41-55, 2013.

MACHADO, Cristiane. Avaliação Externa e Gestão Escolar: reflexões sobre uso dos resultados. Revista@mbienteeducação, v. 5, n. 1, p. 70-82, jan/jun, 2012.

MINHOTO, Maria Angélica Pedra. Notas sobre a avaliação da qualidade de sistemas educacionais. In: BAUER, Adriana; GATTTI, Bernadete A.; TAVARES, Marialva R. (Org.) Vinte e cinco anos de avaliação de sistemas educacionais no Brasil: origem e pressupostos. Florianópolis: Insular, 2013. p. 137-148. 
OLIVEIRA, Lina Kátia Mesquita. Os sistemas educacionais de avaliação e a gestão educacional. Trabalho apresentado no II CONAVE - Congresso Nacional de Avaliação Educacional. Unesp Bauru, 24-26 set. 2012.

SOUSA, Sandra Maria Zákia Lian; OLIVEIRA, Romualdo Portela de. Sistemas estaduais de avaliação: uso dos resultados, implicações e tendências. Cadernos de Pesquisa, v.40, n. 141, p. 793-822, set./dez. 2010.

SOUSA, Sandra Maria Zákia Lian. Avaliação externa e em larga escala no âmbito do estado brasileiro: interface de experiências estaduais e municipais de avaliação da educação básica com iniciativas do governo federal. In: BAUER, Adriana; GATTI, Bernadete A.; TAVARES, Marialva R. (Org.). Vinte e cinco anos de avaliação de sistemas educacionais no Brasil: implicações nas redes de ensino, no currículo e na formação de professores. Florianópolis: Insular, 2013. p. 61-87.

SANTOS, Catarina de A.; NOGUEIRA, Danielle X. P.; JESUS, Girlene R.; CRUZ, Shirleide P. da S. Avaliação de desempenho docente nas redes estaduais de educação básica no Brasil. Trabalho apresentado no III Congresso IberoAmericano de Política e Administração da Educação. Zaragoza. 14 a 17 de novembro de 2012.

VIANNA, Heraldo M. Medida da qualidade em educação: apresentação de um modelo. Estudos em Avaliação Educacional, São Paulo, n.2, p.99-104, jul/dez. 1990.

CRISTIANE MACHADO é doutora em Educação pela Faculdade de Educação da Universidade de São Paulo (FEUSP). Professora titular do PPGE da Universidade Cidade de São Paulo. Coordenador do Grupo de Estudos e Pesquisas em Política Pública, Avaliação e Qualidade (Geppaq). E-mail: cristiane13machado@yahoo. com.br

OCIMAR MUNHOZ ALAVARSE é licenciado em Pedagogia pela Universidade Federal de São Carlos-SP (1984), Mestre (2002) e Doutor (2007) em Educação pela Universidade de São Paulo. Professor da Faculdade de Educação da Universidade de São Paulo. Coordenador do Grupo de Estudos e Pesquisas em Avaliação Educacional (Gepave). E-mail: ocimar@usp.br

PAULO HENRIQUE ARCAS é Licenciado em História pela Universidade de São Paulo (1997). Mestre (2003) e Doutor em Educação pela Universidade de São Paulo (2009). Atualmente é consultor do Instituto Internacional de Planeamiento de la Educación (IIPE - UNESCO). E-mail: arcasmg@uol.com.br

Recebido em abril de 2015

Aprovado em setembro de 2015

680 - RBPAE - v. 31, n. 3, p. 667 - 680 set./dez. 2015 